Revue d'histoire de l'Amérique française

REVUE D.HISTOIRE DE L'AMÉRIQUE FRANÇAISE

\title{
Le Collège-Séminaire de Nicolet (1803-1863)
}

\section{Claude Lessard}

Volume 25, numéro 1, juin 1971

URI : https://id.erudit.org/iderudit/303041ar

DOI : https://doi.org/10.7202/303041ar

Aller au sommaire du numéro

Éditeur(s)

Institut d'histoire de l'Amérique française

ISSN

0035-2357 (imprimé)

1492-1383 (numérique)

Découvrir la revue

Citer cet article

Lessard, C. (1971). Le Collège-Séminaire de Nicolet (1803-1863). Revue d'histoire de l'Amérique française, 25(1), 63-88. https://doi.org/10.7202/303041ar d'utilisation que vous pouvez consulter en ligne.

https://apropos.erudit.org/fr/usagers/politique-dutilisation/ 


\title{
LE COLLEGE-SÉMINAIRE DE NICOLET (1803-1863)
}

\author{
Claude lessard \\ directeur des Archives \\ Université du Québec \\ à Trois-Rivières
}

Après les séminaires de Québec et de Montréal, la troisième institution de notre province qui, en 1803 , se met à dispenser l'enseignement classique est le Séminaire de Nicolet. Les débuts de cette maison d'enseignement sont difficiles car on manque d'argent, de locaux, de personnel, de livres; cela explique dans une large mesure l'irrégularité de l'enseignement jusque vers 1830. A ce moment-là, professeurs et élèves entrent dans le nouvel édifice dont la construction vient d'être terminée. A partir de cette date, les sept classes du cours classique ${ }^{1}$ et la classe française ${ }^{2}$ apparaissent au palmarès tous les ans. Il faut aussi souligner qu'on met alors sur pied une classe d'anglais ${ }^{3}$ qui se transforme plus tard en classe commerciale ${ }^{4}$. Il y a, enfin, des cours de théologie à l'intention des professeurs qui se destinent à la prêtrise. Ces divers cours sont au programme du Séminaire de Nicolet jusqu'à la fin de la période que nous étudions dans le présent travail, c'est-à-dire jusqu'à l'affiliation en 1863. Le Séminaire de Nicolet fut une maison ou s'enseignaient une foule de disciplines. A cause de cela, on peut être porté à croire que l'instruction avait pour les professeurs plus d'importance que la formation morale. Il n'en est pas ainsi toutefois, car les ensei-

${ }^{1}$ Le cours dure huit ans mais il y a sept classes. En effet, jusqu'en 1858 , les élèves de philosophie sont groupés dans une seule classe même si le cours dure deux ans. On y enseigne, une année, la philosophie et les mathématiques et l'autre, la physique et les autres sciences. Certains élèves étudient l'une ou l'autre matière en premier suivant l'année où ils entrent en philosophie. A partir de 1858 , il y a toujours deux classes simultanées de philosophie, avec le même programme qu'avant. Ce qui place la physique et les sciences en philosophie II. J.-A.-I. Douville, Histoire du Collège-Séminaire de Nicolet, 1803-1903 (2 vol., Beauchemin, 1903), I: 417-418.

2 Classe du cours primaire fondée en 1801 et entretenue aux frais du Séminaire de Nicolet. C'est à l'époque la seule école primaire de la paroisse de Nicolet. Voir Douville, op. cit., I: 8, 314-318.

3 On y enseigne seulement l'anglais à tous les élèves divisés en deux groupes. Les cours se donnent en dehors de l'horaire régulier des classes, Directeur à l'évêque, 1804-1816, Archives du Séminaire de Nicolet (désormais ASN), I: 27, 113.

4 A l'anglais s'ajoutent de la comptabilité, du français, de l'arithmétique, du latin, de la géographie, de l'histoire, etc... AAQ, Séminaire de Nicolet, IV, $\mathrm{r}$.

RHAF, vol. 25 , no 1 (juin 1971)

[63] 
gnants sont avant tout des éducateurs qui essaient de transmettre le message non seulement par leurs cours, mais aussi par le règlement et les occupations parascolaires.

Le premier règlement du Séminaire de Nicolet est rédigé par l'évêque de Québec, ${ }^{5}$ qui le remet au directeur. Ce dernier est responsable du choix des programmes des études. Comme les éducateurs québécois du temps ne connaissent que le Ratio studiorum* des Jésuites, ils optent pour ce plan d'études. En complément aux études, il existe, à Nicolet, comme dans les autres collèges, une académie littéraire de discussions dont font partie les élèves les plus âgés ${ }^{6}$. Avec l'Académie, nous entrons dans le domaine des occupations parascolaires que nous décrirons aussi plus en détail dans les pages qui vont suivre. Notre étude se terminera par de brèves observations d'abord sur les éducateurs qui sont alors chargés de ce collège puis sur les élèves et les carrières qu'ils choisissent.

\section{Règlements}

En 1803, quand est fondé le Séminaire de Nicolet, les autorités adoptent le genre de règlement qui leur semble le plus apte à donner une formation solide aux jeunes gens qui sont sous leur tutelle. Le premier texte est de la main de l'évêque de Québec ?, Mgr Denaut. Au début de 1804, il vient lui-même le faire connaître aux éducateurs nicolétains ${ }^{8}$. A ce premier texte, ses suc-

* C'est le nom qui désigne le programme des études des Jésuites depuis 1598; il fut maintenu sans changement jusqu'en 1832.

5 Durant cette période, les auteurs de ces directives furent Mgrs Denaut, Plessis et Signay.

6 Versification à Philo II, i.e. de la 3e à la physique; Gabriel Compayré, Histoire critique des doctrines de l'éducation..., I: 179. A Nicolet, on trouvera tous les renseignements désirés dans les registres de cette société.

7 Nicolet faisait alors partie du diocèse de Québec; il en sera ainsi jusqu'à la fondation du diocèse de Trois-Rivières en 1852, auquel Nicolet sera rattaché. La situation change une dernière fois en 1885 quand est fondé le diocèse de Nicolet.

8 Règlement pour les écoliers, ASN, Séminaire, I: 37, 37b bis; AAQ, Séminaire de Nicolet, I: 10. Copie du document précédent. Il s'agit bien de 1804 , non de 1803, car tout semble indiquer que le mois de janvier 1804 est celui où se fait l'ouverture de l'école latine, et, par conséquent du Séminaire de Nicolet. D'abord le premier règlement est daté du 3 janvier 1804. Les autres faits que l'on pourrait apporter comme preuves sont: instructions pour établir le premier directeur, datées du 11 janvier 1804; octroi d'une pension gratuite à la famille Brassard, décidée le 24 janvier 1804. Voir Mgr J.-A.-I. Douville, Histoire du Collège-Séminaire de Nicolet, I: 17 et ASN, Boîte, III : 9. 
cesseurs en ajoutent d'autres qui le modifient peu: on s'en rend compte en lisant les règles de $1842 .{ }^{9} \mathrm{Au}$ cours de la période qui va de 1803 à 1842, nous avons aussi trouvé des instructions personnelles de Mgr Plessis, en 1813 et en $1818^{10}$, de Mgr Panet le 9 février 1832 et de Mgr Signay le 5 mai $1834^{11}$.

C'est dans ces divers textes que sont énumérées les règles à suivre pour tous ceux qui habitent le collège. Ici nous traiterons surtout du règlement des élèves parce qu'on lui consacre beaucoup d'espace.

Il comporte les chapitres suivants: règlement des pensionnaires et des externes, pour les jours de classes, les dimanches et les jours de congé. Nous trouvons ensuite le règlement des ecclésiastiques et quelques observations sur le costume, le parloir, les sorties, les vacances et l'alimentation. 1863:

Les jours de classe, l'élève suit l'horaire suivant, de 1804 à

$\begin{array}{ll}5 \text { h. ou } 5 \text { h. } 30 & \text { lever } \\ 5 \text { h. } 45 & \text { prière, étude } \\ 7 \text { h. } & \text { déjeuner } \\ 7 \text { h. } 20 & \text { messe, récréation } \\ 8 \text { h. } 10 & \text { classe } \\ 10 \text { h. } & \text { récréation } \\ 10 \text { h. } 15 & \text { étude } \\ 11 \text { h. } 15 & \text { examen, dîner, récréation } \\ 1 \text { h. } & \text { étude } \\ 2-4 \text { h. } & \text { classe } \\ 4 \text { h. } & \text { collation } \\ 4 \text { h. } 30 & \text { étude } \\ 6 \text { h. } 25 & \text { chapelet, lecture spirituelle } \\ 6 \text { h. } 55 & \text { souper, récréation } \\ 8 \text { h. } 30 \text { ou } 8 \text { h. } 15 & \text { coucher 12. }\end{array}$

9 Directions pour les professeurs, AAQ, Séminaire de Nicolet, I: 14; Clauses additionnelles au règlement, AAQ, Séminaire de Nicolet, I: 15.

10 Instructions données par Mgr Plessis au directeur, 12 septembre 1813, AAQ, Séminaire de Nicolet, I: 62; Règlement des ecclésiastiques du Séminaire de Nicolet, 15 avril 1818, ASN, Séminaire, I: 40, 40bis.

11 Clauses additionnelles au règlement donné par Mgr Plessis, ASN, Séminaire, I: 34.

12 ASN, Séminaire, I: 37; les seuls changements à noter en 1863 en regard de 1804 ne sont que des variations de cinq à dix minutes dans les heures de certains exercices. C'est un horaire que nous retrouvons avec peu de changements en 1857 à l'Ecole Normale Laval. Voir André LabarrèrePaulé, Les instituteurs läques au Canada français (1836-1900) (Québec, 1965), 212-213. 
Cet horaire ressemble à celui des séminaires de Québec et de Montréal. Nous remarquons qu'il y a équilibre entre les heures de travail et les moments de détente. Par exemple il y a quatre heures de classe, par jour, et cinq heures d'étude. C'est à peu de chose près ce que nous retrouvons dans les autres institutions du temps.

La lecture de cet horaire nous apprend aussi que la messe a lieu après le déjeûner. Cet état de choses ne doit pas nous surprendre car les élèves ne communient pas sur semaine ${ }^{13}$. Ce même horaire nous a aussi permis de constater que les élèves se couchent tôt. Les éducateurs de ce temps peuvent difficilement faire autrement car on s'éclaire alors à la chandelle, et ce genre d'éclairage n'est pas assez puissant pour chasser complètement l'obscurité. Voilà pourquoi, il vaut mieux s'abstenir d'étudier en soirée.

Ce règlement des jours de classe est l'objet de quelques changements le dimanche. Ce jour-là, il n'y a pas de classe mais les exercices religieux les remplacent comme le montre l'horaire de cette journée:

$\begin{array}{ll}5 \mathrm{~h} .25 & \begin{array}{l}\text { lever } \\ \text { prière, étude en partie prise par les exercices } \\ \text { des cérémonies liturgiques } \\ \text { congrégation, messe } \\ \text { déjeûner, récréation en partie prise par les } \\ 6 \mathrm{~h} .30 \text { ou } 7 \mathrm{~h} .\end{array} \\ 8 \mathrm{~h} . & \begin{array}{l}\text { exercices des cérémonies liturgiques } \\ \text { grand'messe à l'église paroissiale }\end{array} \\ 10 \mathrm{~h} . & \text { dîner, récréation } \\ 11 \mathrm{~h} .30 & \text { catéchisme, récréation } \\ 1 \mathrm{~h} . & \text { vêpres à l'église paroissiale } \\ 3 \mathrm{~h} . & \text { collation, récréation } \\ 4 \mathrm{~h} . & \text { étude } \\ 5 \mathrm{~h} . & \text { lecture spirituelle } \\ 6 \mathrm{~h} . & \text { souper, récréation } \\ 6 \mathrm{~h} .15 & \text { leste comme aux jours de classe } 14 .\end{array}$

On voit que les exercices religieux occupent plusieurs heures du jour. Le premier est la messe dite de communion, car, le dimanche, la communion est permise; puis le deuxième, c'est la grand'messe, à dix heures. Ainsi que leurs confrères de Québec ${ }^{15}$, de Sainte-Thérèse ${ }^{16}$ et probablement d'ailleurs, les pensionnaires

${ }^{13} \mathrm{La}$ communion fréquente ne se répand qu'au début du $20^{\mathrm{e}}$ siècle, sous l'impulsion de Pie X.

14 ASN, Séminaire, I: 37.

15 Ibid., III : 48.

16 Emile Dubois, Le petit Séminaire de Sainte-Thérèse (Montréal, 1925), 257. 
et les externes de Nicolet vont, les dimanches et fêtes, entendre la grand'messe à l'église de la paroisse. L'abbé Jean Raimbault, curé de Nicolet, en même temps que supérieur du Séminaire ${ }^{17}$, insiste auprès des directeurs pour qu'ils maintiennent cette coutume. La raison de cette intervention, c'est que les maîtres n'aiment pas voir leurs élèves s'asseoir près des jeunes filles ${ }^{18}$. Ils n'ont pas gain de cause toutefois. En effet, leurs jeunes gens continuent jusqu'en 1863 à se rendre à l'église paroissiale pour la grand'messe et les vêpres ${ }^{10}$. Parmi les services que rend le Séminaire de Nicolet à la fabrique de Nicolet, nous notons enfin ceux de l'élève qui est sacristain à l'église ${ }^{20}$.

Ces exercices religieux sont ceux dont on parle le plus mais ce ne sont pas les seuls : il y a aussi la récitation de l'office de la Sainte Vierge ${ }^{21}$, la lecture spirituelle et les répétitions des cérémonies liturgiques. Au total, les exercices à caractère religieux du dimanche durent plus longtemps que les cours les autres jours de la semaine. Il semble donc que cette journée permette peu aux élèves de se reposer; ils peuvent le faire les jours de congé.

A Nicolet, les congés sont répartis comme suit: deux aprèsmidi depuis les vacances jusqu'à la Toussaint, un seul après-midi depuis la Toussaint jusqu'à Pâques, un jour entier depuis Pâques jusqu'aux vacances d'été ${ }^{22}$.

Nous avons aussi relevé quatre congés extraordinaires par an : ceux des maîtres, de l'évêque et de son coadjuteur et celui de la sortie du premier janvier ${ }^{23}$. Pour occuper les temps libres de ces journées de repos, les jeux sont rares. Plusieurs directeurs, dont l'abbé Joseph-Onésime Leprohon ${ }^{24}$, font faire des travaux horticoles et des promenades à la campagne ${ }^{25}$.

17 C'est depuis l'année 1806-07 que le dirigeant du Séminaire de Nicolet a le titre de supérieur. De cette date à $1840-41$, ce supérieur est le curé de Nicolet, soit les abbés Jean Raimbault et Jos.-Onésime Leprohon, à qui succède un triumvirat, puis un supérieur en titre après 1847-48, toujours choisi depuis ce temps parmi le personnel du séminaire.

18 ASN, Séminaire, III : 48.

$19 \mathrm{Vu}$ l'exiguïté des locaux du séminaire et sa pauvreté, on ne peut alors se permettre de faire de belles cérémonies religieuses comme à l'église.

20 ASN, Séminaire, I: 39.

21 On la récite presque toujours, même si la première congrégation définitive ne date que de 1823 .

22 ASN, Séminaire, I: 37.

23 ASN, Séminaire, V: 90.

${ }^{24}$ Louis-Edouard Bois, M. Jos.-O. Leprohon (Québec, 1870), 25 ; ASN : Séminaire, III : 59.

25 ASN, Séminaire, I: 37. 
Après le règlement des pensionnaires, le cahier des règles contient celui des externes ${ }^{26}$ qui indique ce qu'ils doivent faire quand ils sont au collège et quand ils sont dans leurs foyers ${ }^{27}$. Voyant dans l'externe un être aussi exposé, sinon plus, que le pensionnaire à succomber à ses mauvais penchants, l'auteur du règlement voudrait que ce dernier mène une vie identique à celle du pensionnaire. En principe, l'externe n'est pas plus libre que le pensionnaire. En pratique, il l'est car un surveillant n'est pas toujours près de lui pour l'empêcher d'agir à sa guise. Il faut aussi rappeler qu'il a peine à suivre son règlement car la vie de famille ne peut pas se régler suivant un horaire aussi uniforme que celui du collège. Le directeur est conscient de ce problème. Voilà pourquoi il invite les parents à collaborer avec lui ${ }^{28}$.

Les ecclésiastiques n'échappent pas aux directives de l'évêque car la plupart sont jeunes et viennent de passer sans transition du statut d'élève à celui de professeur, sans avoir pu profiter de quelques années de grand séminaire. Tout en enseignant et en surveillant leurs adolescents, ils étudient la théologie et s'initient à leur futur rôle de pasteurs d'âmes. L'horaire qu'ils ont à suivre diffère peu de celui des pensionnaires. Il leur faut non seulement se plier à cet horaire mais aussi et surtout exercer une surveillance attentive et continuelle sur leurs jeunes gens. Le directeur les fait aussi prêcher à tour de rôle à la grand'messe paroissiale. L'un d'eux sert cette messe comme thuriféraire ou cérémoniaire. Ce sont eux enfin qui font la leçon dominicale de catéchisme, de même que les exercices de chant et des cérémonies liturgiques. Jusqu'en 1855, leur formation théologique est sous la responsabilité du curé de Nicolet. Par la suite, un prêtre du collège fait ce travail ${ }^{29}$. Le plus souvent, c'est le directeur.

Nous avons remarqué que le supérieur est assez souvent mécontent du petit nombre d'heures que consacrent les ecclésiastiques à l'étude de la théologie. Il doit donc venir de temps à autre leur rappeler l'importance des études théologiques pour leur future carrière. Vu les lacunes dont nous venons de faire état, nous croyons que les succès furent plus apparents du côté moral. En effet, par ces cours, on a formé plus de prêtres à l'âme bien trempée que de théologiens ${ }^{30}$.

Les élèves portaient un costume caractéristique. A Nicolet, le principal élément du costume des élèves, même en 1803, est

${ }^{26}$ Le mot externe s'entend dans le même sens qu'aujourd'hui; cependant, pour être externe, il faut habiter le village de Nicolet.

27 ASN, Séminaire, I : 43.

28 ASN, Séminaire, 1: 43; XXXIX : 6.

29 Douville, op. cit., II : 52.

30 ASN, Séminaire, I: 40. 
un "capot" bleu avec nervures blanches qu'on porte attaché avec une ceinture qui est toujours verte ${ }^{31}$. Ce costume reste le même jusqu'en 1863. Sous peine de punitions pouvant aller jusqu'à l'expulsion, tous les élèves doivent le porter chaque jour ${ }^{32}$.

Après le costume, un chapitre du règlement traite des sorties et des vacances. Nous y apprenons d'abord que les pensionnaires d'alors ne vont presque jamais chez leurs parents durant l'année scolaire. L'un des rares endroits où ces derniers peuvent rencontrer leurs enfants est au parloir du collège ${ }^{33}$. Ces visites sont inscrites dans un cahier ${ }^{34}$, qui sert à renseigner le directeur. Aucune fille ni femme ne peut aller ailleurs qu'au parloir ${ }^{35}$.

Les dimanches et les jours de grande fête, il est permis aux parents de sortir avec leurs enfants depuis les vêpres jusqu'au coucher. Le directeur leur interdit cependant d'aller au-delà des limites de la paroisse de Nicolet sous peine de sanctions ${ }^{36}$. Cette sortie dominicale est la seule qu'on autorise sauf pour les élèves qui sont atteints de maladies contagieuses ou qui mouillent leur lit ${ }^{37}$.

C'est donc dire que les élèves vont peu à l'extérieur de la clôture du collège, mais ils ne semblent pas vouloir sortir plus souvent. Une fois l'année scolaire terminée, ce sont les vacances d'été. Nous dirons peu de choses des vacances du Jour de l'an ${ }^{38}$ car elles durent à peine quelques jours. En effet, en 1804, Mgr Denaut accepte que les pensionnaires sortent le lendemain ou le surlendemain du Jour de l'an ${ }^{39}$. Son successeur, Mgr Plessis, supprime cette sortie ${ }^{40}$, mais Mgr Signay la rétablit en 1842 à la demande de l'abbé J.-B.-A. Ferland ${ }^{41}$. En raison des nombreux retards au retour, cette journée de sortie disparaît peu après jusqu'en $1891^{42}$.

Les documents font beaucoup plus mention des vacances d'été car les éducateurs voudraient que l'évêque en modifie les

31 Ibid.: 37.

32 Ibid.

33 Ibid.: 37, 37 bis; ASN, Séminaire, III : 59.

34 ASN, Boîte, IV (Cahier du parloir, 1819 à 1827).

35 ASN, Séminaire, I : $39 ; 9$.

36 Ibid., I : 39.

37 Ibid.

38 Ibid.

39 ASN, Séminaire, I: 37.

40 Wilfrid Lebon, Histoire du Collège de Sainte-Anne-de-la-Pocatière (2 vol., 1948-1949), I: 105.

41 ASN, Séminaire, I : 37.

42 Ibid. 
dates ${ }^{43}$. Elles ont d'abord lieu entre le 15 août et le premier octobre. Après 1850, les dates de ce moment de repos se rapprochent un peu de celles d'aujourd'hui, c'est-à-dire le 15 juillet et le $1^{\text {er }}$ septembre ${ }^{44}$.

Le règlement ne dit rien des punitions. Les autres documents que nous avons consultés n'en parlent pas non plus. Par contre, au sujet de l'alimentation, nous sommes mieux renseignés. Nous savons par exemple que la façon d'agir des premiers procureurs, dont l'abbé Harper, consiste à bien nourrir les élèves durant les premières semaines de l'année et à donner par la suite des mets moins appétissants. Cette méprisable habitude dure jusque vers 1845. A ce moment-là, l'abbé Ferland, qui est au collège depuis $1841^{45}$, fait observer que cette manière d'agir est la cause d'une diminution des élèves ${ }^{46}$. Il met $\mathrm{Mgr}$ Signay au courant de cet état de choses. Ce dernier y remédie tant bien que mal en remettant un menu plus équilibré ${ }^{47}$. Ce changement et l'adoption du système des quart-pensionnaires ${ }^{48}$ (pensionnaires prenant un repas au séminaire) semblent expliquer qu'il y ait par la suite une augmentation assez marquée de la population étudiante ${ }^{49}$.

A la suite de ce que nous venons de révéler sur les cadres de la vie étudiante au Séminaire de Nicolet, dans la première moitié de $\mathrm{XIX}^{\mathrm{e}}$ siècle, nous ne pouvons dire que ceci: le règlement est toujours le même et la discipline est sévère. Il ne peut en être autrement car, comme l'éducation dispensée dans un collège a pour but de préparer des jeunes gens à vivre dans la société dont ils font partie, il faut que les maîtres fassent appliquer un règlement qui convienne à cette société dans laquelle ils se trouvent. $\mathrm{Or}$, au $\mathrm{XIX}^{\mathrm{e}}$ siècle, à Nicolet comme ailleurs, les jeunes gens vivent en chrétienté rurale, donc dans une société homogène et fortement hiérarchisée; c'est pourquoi le règlement d'alors et

43 Surtout l'abbé J.-0. Leprohon et des prêtres du Collège de Ste-Annede-la-Pocatière auxquels se sont joints d'autres du Séminaire de Québec. ASN, Séminaire, V: 88, 89; Wilfrid Lebon, ibid., I: 105.

44 Des renseignements à ce sujet peuvent être retracés dans une boîte métallique $\left(n^{\circ} 12\right)$ où se trouvent conservés aux archives du Séminaire de Nicolet les ordos et les journaux de classe de la période qui nous intéresse actuellement.

45 Douville, op. cit., II: 18. Il avait été élève, puis ecclésiastique au Séminaire de Nicolet. Il fut aussi professeur alors qu'il était séminariste.

46 Douville, op. cit., I: 320.

47 Ibid., 319.

48 Ibid., 334, 339.

49 Ibid., 339. 
la forte discipline qui en est l'épine dorsale ont leur place pour former les futurs hommes de cette société ${ }^{50}$.

\section{Programme d'études}

L'influence des temps passés, que nous avons signalée lors de nos commentaires sur le règlement, se manifeste à nouveau dans le programme des études. En effet, le système d'enseignement qui est suivi à Nicolet est en somme le même que celui des collèges de Québec et de Montréal. S'il en est ainsi, c'est que les premiers directeurs viennent de ces maisons, surtout de Montréal ${ }^{51}$. Ces derniers appliquent leurs propres méthodes de travail, mais les directives qu'ils suivent et les volumes qu'ils utilisent viennent de Québec, de l'évêque généralement. Dans ces deux collèges de Québec et de Montréal, les professeurs ne connaissent que le programme d'études des Jésuites, sauf qu'à Québec certains utilisent les traités de Batteux et de Rollin ${ }^{52}$. A Nicolet, personne ne semble connaître ces auteurs. Nous avons remarqué aussi qu'aucun éducateur nicolétain n'est au courant des problèmes pédagogiques dont on discute avec force en France depuis J.-J. Rousseau. Selon eux, la solution de la sagesse est d'ignorer tout ce qu'on dit alors en Europe contre les études gréco-latines et de regarder la liste des grands hommes qui se sont formés en étudiant les disciplines mentionnées dans le Ratio ${ }^{53}$.

Pour donner une description du cours d'études au Séminaire de Nicolet, nous énumérerons d'abord la liste des disciplines qui y furent enseignées, puis nous verrons comment les éducateurs nicolétains parviennent à les faire connaître même s'ils ont peu de livres. En effet, ils rencontrent beaucoup de difficultés pour se procurer des manuels et des instruments scientifiques. Quand ils n'en trouvent pas, ils font usage des notes manuscrites de leurs prédécesseurs. Ainsi en est-il jusqu'en 1836. Par la suite, le manuscrit cède la place à l'imprimé et des instruments scientifiques sont achetés en Europe.

${ }^{50}$ Le règlement dans les collèges, 33 (Réunion annuelle des directeurs d'élèves, 19-20 octobre 1960).

51 Ce fut le cas de l'abbé Jean-Baptiste Roupe (1804-1806) et de l'abbé Joseph-Onésime Leprohon (1816-1841). Voir pour plus de détails: Antoine Letendre, éd., Album-souvenir du $150^{e}$ anniversaire de fondation du Séminaire de Nicolet, 24.

52 Lionel Groulx, L'enseignement français au Canada (2 vol., Montréal, 1931, 1933), I: 114 .

53 Comme nous n'avons rien trouvé sur ce sujet durant les premières années du séminaire et ce après six ans de recherches, nous croyons avoir raison de faire une affirmation aussi catégorique. 
Un certain nombre d'écrits ${ }^{54}$ nous portent à croire que le Séminaire de Nicolet adopte le Ratio studiorum ${ }^{55}$ comme programme d'études. Quelques transformations y sont faites surtout après 1830, mais l'étude des langues anciennes a toujours la première place.

N'oublions pas cependant ${ }^{56}$ que le français est aussi enseigné de la sixième à la seconde, mais avec une lenteur telle que les humanistes ${ }^{57}$ étudient encore la grammaire ${ }^{58}$. Après 1836, toutefois, l'étude de la grammaire française s'arrête souvent en troisième. C'est sans doute dû au fait que les élèves d'alors font plus de travaux pratiques qu'avant. En effet, en troisième, en seconde et en première, ils rédigent des interprétations et des amplifications.

En latin, les professeurs nicolétains copient assez médiocrement les habitudes de leurs prédécesseurs. Incapables de revenir au système des prélections ${ }^{59}$ comme le faisaient les Jésuites, ils concentrent leurs travaux sur l'étude de la grammaire. Gardons-nous de croire cependant que les professeurs ne donnent jamais d'exercices, de thèmes, de versions et d'explications des auteurs. La progression qu'ils mettent dans les travaux appelle sans doute des réserves mais la liste que nous allons donner va nous révéler des méthodes dont la recette ne s'est pas perdue:

éléments: grammaire, thèmes, versions.

syntaxe: grammaire, thèmes, versions, explications de dialogue et de fables.

méthode: grammaire, thèmes, versions, compositions, prosodie.

54 (Anonyme), L'enseignement classique dans le Québec, 5; Groulx, op. cit., I: 114, 194; Olivier Maurault, Le petit séminaire de Montréal, (Montréal, 1918), 70; Le Canadien, 24 septembre 1831, 1s; Directeur à l'évêque, ASN, I: 404; J.-B.-A. Ferland, "Mgr J.-O. Plessis", Foyer canadien, I (1863) : 78 .

55 Voir p. 64.

56 Ce sont là les seules sources d'information qui nous aient permis de retracer assez bien ce programme d'études. On peut trouver ces renseignements dans les registres intitulés "Palmarès" et d'autres dont les titres commencent par "Lettres de ..."

57 La classe d'humanités doit être la seconde ou belles-lettres. Mais, au Séminaire de Nicolet, durant les vingt premières années surtout, l'on a tellement groupé ensemble les élèves de troisième et de seconde que l'on peut avoir raison de donner ce nom pour désigner l'une ou l'autre classe.

58 Directeur à l'évêque, 1804-1816, ASN, I: 27.

59 François de Dainville, La naissance de l'humanisme moderne (Paris, 1940), 234. 
versification: grammaire, versions, thèmes, récitation, explication de vers, compositions.

belles-lettres: grammaire, versions, thèmes, compositions, vers latins, discours.

rhétorique: amplifications, versions, thèmes, vers latins ${ }^{60}$.

Au nombre des langues au programme des études, il y a aussi le grec mais il met bien du temps à se faire accepter des éducateurs nicolétains. Nous croyons que les premiers professeurs ne l'enseignent pas, bien qu'il soit officiellement au programme depuis la fondation du séminaire. Les palmarès ne signalent aucun prix en cette matière avant 1837 . Cette dernière constatation ne nous permet pas d'affirmer qu'on ne l'enseigne pas alors. Cependant nous savons par la correspondance du temps que l'intérêt que portent les Nicolétains à cette discipline n'est que passager pendant les trente-cinq premières années de cette institution. Au cours de cette période, il semble y avoir des cours de grec quand passe au collège un professeur qui a quelques connaissances de cette langue. En pareil cas, il arrive que les élèves de philosophie suivent ses cours. Si l'enseignement du grec a peine à se maintenir, l'une des causes est sans doute le manque de manuels car l'abbé Leprohon ne semble pas en avoir trouvé. Quoi qu'il en soit, ces observations ne sont que des hypothèses du moins jusqu'en 1837-39, années où le grec apparaît dans les classes allant d'éléments à la rhétorique ${ }^{61}$. Les travaux se ressentent de ces difficultés car, avant 1836 , le collège reçoit ${ }^{62}$, échange ou achète peu de morceaux choisis des classiques grecs ${ }^{63}$.

60 ASN, Séminaire, I: $39 ; 16$.

61 Les difficultés que nous avons rencontrées à préciser les débuts des cours de grec à Nicolet, on les rencontre pour d'autres collèges. Ainsi, Mgr Arthur Maheux souligne qu'il est assez difficile de préciser "la date de l'introduction du grec dans le collège classique du Québec. Les documents n'en parlent pas". Par ailleurs, parmi les idées qui ont amené à mettre le grec au programme, il y voit une influence extérieure car, dit-il, "les collèges américains enseignaient le grec, c'était une vieille tradition des universités de Grande-Bretagne. Les Anglais venus à Québec et à Montréal après la cession du Canada à l'Angleterre, que ce fût d'Angleterre ou des EtatsUnis, apportaient ces traditions. C'est par ces réclames publiées dans les journaux canadiens, par les instituteurs privés qu'on peut s'en rendre compte... Ainsi on annonce le grec; $y$ eut-il des élèves pour mordre à l'appât... De toute façon, la seule annonce suffisait à bien placer les instituteurs anglais dans l'opinion publique. Il $\mathrm{y}$ avait là une menace pour le prestige de nos collèges classiques et on conçoit leur désir de relever cette sorte de défi." Rapport de la commission du programme de la Faculté des Arts (1957-60), I: $124 \mathrm{~s}$.

62 Un certain nombre de gens, surtout des prêtres, lèguent à leur mort leur bibliothèque au Séminaire de Nicolet.

63 ASN, Séminaire, I: 66 à 75. 
L'enseignement de l'anglais, d'abord négligé, débute vers 1810. Il importe de signaler que ces cours ne sont pas à l'horaire régulier des classes. En effet, jusque vers 1820, ils se donnent uniquement les jours de congés à tous les élèves à la fois et par un seul professeur. Ce système donne peu de résultats. Alors, on décide, probablement après 1820, de mettre l'anglais au programme régulier des six premières années du cours classique. Cette innovation permet d'introduire définitivement des leçons de grammaire, des versions et des thèmes.

Après les langues, le programme mentionne l'histoire et la géographie. En histoire, les cours commencent, semble-t-il, en syntaxe et se poursuivent jusqu'en philosophie. Dans la classe de cinquième, le programme est le suivant: histoire sainte, histoire du Canada ${ }^{64}$ et de temps à autre, mythologie ${ }^{65}$. En quatrième, des cours d'histoire du Canada sont encore au programme parce que certains élèves arrivent directement de la sixième ${ }^{66}$; on $\mathrm{y}$ enseigne aussi l'histoire ancienne. Quand les élèves de quatrième n'ont pas terminé l'étude de l'antiquité à la fin de l'année, ils la poursuivent en troisième. Dans cette classe, durant la majeure partie de l'année, le professeur enseigne l'histoire moderne ${ }^{67}$. Nous manquons de renseignements pour les classes supérieures, sauf qu'au temps de l'abbé Ferland (1841-1850) un effort est fait en faveur de l'histoire du Canada auprès des jeunes gens plus âgés. Il leur fait lire des textes historiques ${ }^{68}$ et rédiger des travaux à l'Académie pour qu'ils s'intéressent davantage aux hauts faits des temps passés ${ }^{69}$.

En géographie, par contre, les cours portent sur tous les pays du monde et servent presque uniquement à développer la mémoire des élèves. Ils peuvent convenir aux élèves de sixième et de cinquième, mais pas à ceux de troisième et de seconde à qui on aurait pu éviter d'enseigner de telles banalités.

${ }^{64}$ AAQ, Séminaire de Nicolet, I: 14.

65 Directeur à l'évêque, 1804-1816, ASN, I: 14.

$66 \mathrm{Il}$ arrive fréquemment durant les premières années du collège que les élèves sautent des classes. C'est une habitude assez répandue quand, par exemple, les méthodistes n'ont pas assez ou ont trop de connaissances pour monter en troisième. Dans un pareil cas, les élèves faibles restent en méthode et les élèves forts passent en seconde. Nous trouvons de pareils cas dans toutes les classes surtout de 1803 à 1825. Directeur à l'évêque, 1804-1816, ASN, I: 293, 307, 350, 487, 497.

67 ASN, Séminaire, X: 4.

68 Ibid.

69 Registre de l'Académie, ASN, I. 
Les mathématiques sont toujours au programme, mais jusqu'en première les professeurs ne font pas autre chose que d'initier leurs élèves aux règles générales. Durant toute la période que nous étudions ici, la section des mathématiques qui reçoit le plus d'attention dans les basses classes est l'arithmétique. A mesure que l'élève gravit les degrés de son cours classique, les mathématiques perdent de l'importance. En effet, en seconde et en première, elles cèdent assez souvent la place à des leçons de préceptes littéraires. Après la rhétorique, une année est réservée à peu près uniquement à la philosophie et aux mathématiques. C'est donc à ce moment-là qu'elles sont étudiées avec le plus de soin. Pour en savoir davantage sur le contenu des cours, nous laisserons le professeur de philosophie de 1810 nous indiquer ce qu'il enseigne cette année-là :

Ils ont vu les mathématiques savoir: l'arithmétique tout entière, l'algèbre, les raisons, proportions et progressions, tant géométriques qu'arithmétiques, le calcul des radicaux, l'extraction des racines, les équations du premier et du second genre; ils ont résolu sur ces différentes branches de mathématiques quatre-vingts et quelques problèmes ${ }^{70}$.

Entre 1835 et 1840 , une amélioration commence à se manifester dans l'enseignement de cette matière en raison de l'impression de nouveaux manuels d'arithmétique ces années-là. Voilà sans doute pourquoi les élèves sont mieux préparés que leurs prédécesseurs aux "hautes mathématiques" que l'on enseigne en philosophie.

En troisième, en seconde et en première, plusieurs heures sont consacrées chaque semaine aux préceptes littéraires. Le programme est le suivant: en troisième, préceptes de versification; en seconde, ceux de belles-lettres, et en première, ceux de rhétorique. Avant 1833, les cours de préceptes sont donnés de façon irrégulière en raison des classes de seconde et de première qui sont inexistantes certaines années ${ }^{71}$. Il faut aussi souligner que les cours sont dictés et malheureusement difficiles à comprendre. Le Séminaire de Montréal remédie à cette lacune en 1836 quand un de ses professeurs publie un recueil de préceptes littéraires. Par la suite, les directeurs des études ${ }^{72}$ notent une amélioration dans l'enseignement de cette matière.

${ }^{70}$ Directeur à l'évêque, 1804-1816, ASN, I: 148.

71 Aux années suivantes seulement $(1817,1819,1822,1824,1825,1828$, $1831,1833)$ il $y$ a classe en seconde. En première, entre 1815 et 1833, il n'y a classe qu'en 1818 et 1829 .

72 Nous disons directeurs d'études mais ce terme désigne autant les directeurs disciplinaires que les préfets car avant 1840 le directeur cumule les deux charges. 
Quand les élèves passent par chacune des six premières classes du cours classique, ils suivent normalement le programme que nous venons de voir. S'ils subissent avec succès leurs examens de première, ils sont acceptés en philosophie où le programme est celui-ci: philosophie, mathématiques et sciences. En philosophie, les études durent un an. Le contenu des cours est puisé dans des notes manuscrites jusqu'en 1835 et par la suite dans des manuels. C'est ce qui rend possible la permanence de ces cours entre $18088^{73}$ et 1863.

Jusqu'à l'arrivée de l'abbé François Lesieur-Désaulniers, c'est-à-dire jusqu'en 1830 , les sciences font souvent partie du programme de philosophie. A ce moment-là, sous l'influence de l'abbé John Holmes du Séminaire de Québec ${ }^{74}$ et à la suite d'un voyage d'études à Georgetown ${ }^{75}$, l'abbé Désaulniers détache celles-ci de la philosophie. Il reprend aussi la rédaction de ses notes de cours mais le titre est toujours physique. Après 1840 cependant, son programme de physique comporte des notions de minéralogie, de géologie et d'astronomie. Au cours de la même période, les "physiciens" commencent à faire des expériences en laboratoire grâce à l'achat d'un cabinet de physique ${ }^{76}$.

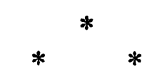

Le programme que nous venons de faire connaître est celui des classes du cours classique dans la première moitié du $\mathrm{XIX}^{\mathrm{e}}$ siècle. Nous n'avons pas tout dit car il se trouve aussi certaines matières que l'on enseigne en dehors des cours réguliers. C'est le cas par exemple du catéchisme qui, au cours du directorat de l'abbé Leprohon, est expliqué chaque jour ${ }^{77}$. L'abbé Leprohon s'intéresse aussi au chant, convaincu qu'il est important dans une maison d'enseignement. C'est l'abbé Jean Raimbault qui l'enseigne le dimanche ${ }^{78}$. A partir de 1818, un régent le remplace ${ }^{79}$ probablement jusqu'en 1833, date de la nomination d'un professeur laïc de musique, Joseph-Videu Hunt ${ }^{80}$. Ce dernier initie les

${ }^{73}$ La première classe de philosophie a lieu au cours de l'année scolaire de 1808-1809.

${ }^{74}$ Ce dernier donne par lettres des conseils sur l'utilisation d'appareils scientifiques et sur la manière d'enseigner certaines parties de la physique.

75 Douville, op. cit.

76 ASN, Séminaire, V: 86.

77 Bois, op. cit., 44.

78 Directeur à l'évêque, 1816-1839, ASN, II : 441.

79 Ibid.: 482.

80 Douville, op. cit., I: 44. 
élèves au solfège, et, fait nouveau, à la musique instrumentale. Ce professeur de musique songe en outre à former une fanfare. Le projet est repris par ses successeurs et c'est l'un d'eux, L.-E. Desjardins ${ }^{81}$, qui réussit en 1854 à faire acheter les instruments essentiels à un tel ensemble musical. Ces améliorations sont toutes faites quand arrive Edmond-Octave Hardy-Chatillon, en $1862{ }^{82}$. En même temps que L.-E. Desjardins, M. Sarony enseigne le dessin ${ }^{83}$. Il donne ses cours de 1851 à 1855.

Outre les classes du cours classique, des professeurs sont chargés de la classe française jusqu'à l'arrivée des Frères des Ecoles chrétiennes en $1887^{84}$. Cette classe est à l'origine du collège ${ }^{85}$ et son programme est semblable à celui des autres écoles primaires du temps. Devant l'impossibilité où nous sommes d'en dire plus long sur ce sujet, nous porterons notre attention sur la classe anglaise. Cette classe, communément appelée classe anglaise ou classe commerciale, permet aux jeunes gens qui ont complété leurs études à la classe française d'acquérir une formation commerciale s'ils ne désirent pas se mettre à l'étude du latin et du grec. Ainsi que nous allons le voir maintenant, le programme est établi en fonction de ce but:

Mathématiques: arithmétique commerciale, algèbre, géométrie, arpentage et navibation;

Tenue de livres: par simple et double entrée;

Grammaire anglaise avec exercices d'orthographe, d'analyse et de composition;

Géométrie élémentaire;

Eléments de l'histoire sacrée, du Canada et d'Angleterre;

Ecriture, lecture et épellation 86 .

\section{Matériel d'enseignement}

Dans les pages précédentes, nous avons parfois mentionné que l'enseignement s'améliore entre 1830 et 1840 . C'est sans doute dû à l'arrivée d'imprimés en plus grand nombre à partir de cette décennie. L'énumération que nous allons maintenant faire du matériel en usage va nous permettre de constater ces améliorations.

\footnotetext{
81 Ibid., II : 65.

82 Ibid., I : 413.

83 A Montréal il faut attendre 1842; Maurault, op. cit., 93.

$84 \mathrm{~A}$ ce moment-là, elle devient une classe préparatoire; voir les Annuaires de la fin de $\mathrm{XIX}^{\mathrm{e}}$ siècle; Douville, op. cit., I: 315.

85 Elle fut donnée en 1801 et c'est de sa transformation qu'est né le collège en 1803 .

86 AAQ, Séminaire, IV : 4.
} 
Les notes manuscrites sont en usage pour les cours suivants: préceptes, philosophie, histoire ${ }^{86 a}$, géographie, physique et architecture. Beaucoup de ces cahiers de notes manuscrites sont de la main de l'abbé Leprohon qui les avait rédigées alors qu'il étudiait au petit Séminaire de Montréal. Ce dernier écrit en 1836 que les professeurs de Nicolet utilisent encore ses notes ${ }^{87}$ pour les matières suivantes: préceptes de versification ${ }^{88}$, de belleslettres et de rhétorique, philosophie, y compris la physique et la mythologie. Les notes manuscrites appartenant à l'abbé Leprohon sont toutes des traités complets. De là vient l'intérêt qu'on leur porte aussi longtemps que les imprimés ne les remplacent pas. Ce qui se réalise après 1835 .

En histoire, on fait aussi usage de notes manuscrites avant 1835. En 1822, nous savons que Charles Wolff copie tous ses cours d'histoire romaine; d'autres élèves font de même en 1823 pour l'histoire moderne. Les archives du séminaire possèdent aussi un résumé de chronologie fait par Louis-Théophile Fortier qui étudie à Nicolet entre 1815 et 1823. En géographie, quelques élèves écrivent aussi leur cours. Louis-Théophile Fortier est encore de ce nombre. Les manuscrits d'histoire font place vers 1830 à des abrégés imprimés spécialement pour le Séminaire de Nicolet. En géographie, les notes manuscrites sont remplacées par des imprimés vers 1828. Cette année-là, James Lane lance sur le marché un manuel de géographie à l'intention des élèves du Séminaire de Nicolet ${ }^{89}$. En physique, les élèves rédigent leurs cours à même les notes de leur maître, jusqu'en 1835. Le professeur utilise les manuscrits de l'abbé Leprohon. Après cette date, l'abbé Désaulniers en recompose un nouveau qui sert encore en 1863.

Quand débutent les cours d'architecture, il n'y a au collège de Nicolet que les cours de l'abbé Jérôme Demers. C'est une copie qui vient d'être faite spécialement pour le Séminaire de Nicolet à la demande de Mgr Signay. Ce cahier est en usage de 1840 à 1863. nologie.

86a Parfois les notes d'histoire ont comme titres: mythologie ou chro-

87 Ibid., V: 82; nous avons pu confirmer la chose par la découverte de notes d'élèves des années d'avant 1836: Louis-Théophile Fortier, CharlesPierre Huot, Julien Perrault, Charles Harper.

88 Les notes sur les préceptes de versification appartiennent à l'abbé Leprohon mais furent faites par son frère Bernard, alors qu'il étudiait au petit Séminaire de Montréal vers $\mathbf{1 8 1 0}$.

89 Géographie à l'usage du Séminaire de Nicolet (Montréal, James Lane, 1828), $49 \mathrm{p}$. 
Ce n'est pas par plaisir que les professeurs recourent aux notes manuscrites. Ils y sont forcés en raison du manque de manuels pour enseigner certaines disciplines. Maîtres et élèves essaient d'en tirer le plus de profit possible tant que les imprimés n'apparaissent pas en plus grand nombre et à prix modéré. Une première amélioration se dessine avec l'arrivée des premiers manuels achetés en Europe ${ }^{90}$.

Au même moment, c'est-à-dire vers 1840 , des manuels canadiens commencent à paraître en plus grande quantité qu'avant. Des imprimés viennent aussi de ceux qui en possèdent ${ }^{91}$. Ceux qui en fournissent le plus sont les évêques de Québec mais leurs livres sont anciens. Un certain nombre en effet ont déjà servi au collège des Jésuites avant $1760^{92}$.

Après consultation de la liste des achats, nous croyons pouvoir affirmer que les grammaires figurent souvent dans les commandes. C'est ce genre de manuels que les libraires canadiens vendent le plus, qu'il s'agisse des grammaires latines ou francaises de Lhomond ${ }^{93}$ ou de celles du petit Séminaire de Montréal ${ }^{94}$. Après 1850, de nouvelles grammaires sont achetées. La plus connue est celle d'Emile Lefranc ${ }^{95}$. Retenons toutefois que les volumes de Lhomond restent encore en usage. On constate par exemple que la grammaire française de cet auteur sert à des élèves nicolétains pendant près de cent ans ${ }^{96}$. D'autres manuels semblables apparaissent après 1840 ; nous mentionnerons ici ceux de Noël et Chapsal ${ }^{97}$, de Lévizac ${ }^{98}$, de Restaut ${ }^{99}$ et celui qui a pour titre Grammaire française pour servir d'introduction à la grammaire latine. En grec, les cours ont peu de valeur, vu

${ }^{80}$ L'année 1836 marque le début de ces achats en Europe par l'abbé Jean Holmes; le tout se continuera à un rythme plus accéléré, croyons-nous, même si aucun document ne nous en donne la preuve directe.

91 Dans les cahiers de lettres du directeur, il parle de démarches qu'il a faites auprès de gens qui ont des volumes.

92 Il est très difficile de préciser le nombre de ces livres; mais nous en avons trouvé plusieurs avec à l'intérieur l'identification de ce collège.

93 Grammaire latine (Paris, Didier, 1835), 70 p. Lhomond était utilisé en 1803 mais nous ne savons où sont rendus les volumes de cette période.

94 Grammaire latine (Montréal, Lowell et Gibson, 1847), 140 p.

95 Grammaire latine (Paris, Jacques Lecoffre et Cie, 1846), $463 \mathrm{p}$.

96 Le révision de ces manuels les a ainsi gardés à la page.

97 Grammaire française (2 vol., Paris, Maire-Nyon, 1860 et 1862), 220 p. et $194 \mathrm{p}$.

98 Grammaire philosophique et littéraire (Paris, Rémont, 1822), $431 \mathrm{p}$. 99 Grammaire française (Paris, Lottin, 1774), $642 \mathrm{p}$. 
que le professeur a peine à faire comprendre le manuel compliqué qu'il utilise. C'est celui de J. B. Gail ${ }^{1}$. Depuis 1838 cependant, la grammaire grecque du petit Séminaire de Montréal remplace ce dernier utilisé en troisième pendant environ dix ans ${ }^{2}$. A l'occasion, les hellénistes travaillent dans les volumes de J.-L. Burnouf, d'Edw. Wettenhal, de Moor et de Nicolas Clénard ${ }^{3}$.

En langue anglaise l'étude des rudiments se fait d'abord dans le manuel de Siret ${ }^{4}$. Jusqu'en 1863, cette grammaire sert toujours à quelques élèves, bien qu'elle ne soit utilisée officiellement comme manuel que de 1803 à 1830 environ. Outre ce manuel français, on fait usage vers 1840 de celui de Lindley Murray ${ }^{5}$.

Pour l'étude des langues, nous voyons les élèves faire des thèmes et des versions. Ils possèdent, pour faire ces travaux, des recueils de morceaux choisis. En latin, Mgr Plessis en remet 150 environ en $1815^{6}$. De cette date à 1836, l'abbé Leprohon en achète à Montréal 70 autres et, en 1836, l'abbé John Holmes en rapporte près de 300 d'Europe $^{7}$. Il semble que par la suite des achats semblables soient faits en Europe à intervalles réguliers. Les professeurs et les élèves ne sont pas mieux servis en extraits d'auteurs grecs car les premiers achats de ce genre ne sont probablement pas antérieurs à 1836, année où l'abbé Holmes en rapporte 112 exemplaires d'Europe. En langue anglaise, un certain nombre des volumes en usage viennent des Etats-Unis. Un inventaire sommaire ${ }^{8}$ nous a permis de découvrir les suivants: Murray Reader, Murray spelling Book, Catholic School Book, English Reader. Beaucoup de ces livres servent de 1820 à 1863. Pour étudier la littérature française, les maîtres n'ont en main que des synthèses peu valables qui racontent

1 Grammaire grecque (Paris, Auguste Delalain, 1818), $284 \mathrm{p}$.

2 Grammaire grecque (Montréal, John Jones, 1837), 321 p.

3 J.-L. Burnouf, Méthode pour étudier la langue grecque (Paris, Auguste Delalain, 1829), 336 p.; Edward Vettenhel, Graeca grammatica (Londres, Thomas Ewing, 1769), 92 p.; Jacques Moor, Elements linguae graecae (Edinbourg, Bell et Bradgute, 1814), 296 p.; Clénard, Grammaire grecque (Paris, Simon Bénard, 1686), 196 p.

${ }^{4}$ Grammaire anglaise (Paris, Beaudry, 1852), 210 p. Nous n'avons pas trouvé d'exemplaires d'éditions antérieures.

ธ English Grammar (Montréal, Armour et Ramsay, 1840), 2141 p.

6 Directeur à l'évêque, 1804-1816, ASN, I: 313s; Ces extraits sont des auteurs suivants: Graelus, Cornelius, Ovide, Cicéron, Virgile, Horace, César, Quinte-Curce, Sénèque, Tite-Live, Phèdre, Salluste, Tacite, Térence, Martial, Flore et Papinius.

7 ASN, Séminaire, V: 85, 86.

8 Impossible de faire plus car il n'existe aucune liste de ces manuels. ASN, Séminaire, I: 66 à 75. 
uniquement la vie des écrivains. La bibliothèque possède peu d'exemplaires de leurs œuvres et l'abbé Holmes n'en achète que quelques-uns en $1836^{\circ}$. C'est donc dire que les éducateurs nicolétains portent une faible attention aux auteurs français. Pour ces raisons, il n'y a pas lieu de nous surprendre de ce que les professeurs utilisent la littérature de La Harpe ${ }^{10}$ entre 1803 et 1863.

Pour l'histoire universelle, les premiers manuels en usage sont des imprimés de Thomas Cary en 1832 et ne traitent que de l'antiquité ${ }^{11}$. L'abbé Ferland ne les aime pas parce qu'ils ne disent rien du Moyen Age et des Temps Modernes. C'est ce qui l'incite à corriger cette lacune. Il y réussit assez bien puisqu'il achète les volumes de l'abbé Drioux et d'Emile Lefranc ${ }^{12}$ qui restent en usage au moins cinquante ans. L'abbé Ferland cherche aussi à trouver en histoire du Canada d'autres manuels que ceux de Charlevoix ${ }^{13}$ et de Joseph-François Perrault ${ }^{14}$. Le volume de F.-X. Garneau lui donne en partie satisfaction mais il s'en sert uniquement pour encourager l'étude de notre histoire ${ }^{15}$.

Dans les premiers livres de géographie en usage: Méthode abrégée pour apprendre la géographie ${ }^{16}$, utilisé en 1815, Géographie à l'usage du Séminaire de Nicolet ${ }^{17}$, et Tables de géographie ancienne et moderne ${ }^{18}$, utilisé en 1829 , les cartes et les exposés bien faits sont rares. La valeur de ces manuels est largement dépassée par celui de l'abbé Holmes: Nouvel abrégé de géographie moderne suivi d'un petit abrégé de géographie ancienne à l'usage de la jeunesse ${ }^{19}$. Après 1831 , cet abrégé devient le livre de base pour les cours de géographie jusqu'au dernier quart du XIX $\mathrm{XI}^{\mathrm{e}}$ siècle.

9 Ibid., V : 85, 86.

10 Abrégé du cours de littérature (Hambourg, s.e., 1800), $246 \mathrm{p}$.

11 Histoire romaine (Québec, Thomas Cary et Cie, 1832), 272 p.

12 Drioux, ptre, Histoire romaine (Paris, Eugène Belin, 1860), 300 p. Nous avons trouvé plusieurs exemplaires de ce manuel. Ici apparaît la première édition trouvée. Emile Lefranc, Histoire ancienne. Histoire romaine. Histoire du Moyen Age. Histoire moderne. Histoire de France (Paris, Jacques Lecoffre, 1846, 1851, 1850, 1846, 1855), 511, 558, 284, 516 et 382 p.

13 BRH, LII (1946) : 302.

14 Abrégé de l'Histoire du Canada. Publié de 1832 à 1836, BRH, LII (1946) : 302s.

15 Groulx, op. cit., I : 203 ; Douville, op. cit., I :295.

16 L'auteur est inconnu (Paris, Bellin, 1793), 614 p.

17 L'auteur est inconnu (Montréal, James Lane, 1828), 49 p.

18 Nous n'avons rien trouvé de plus.

19 (Québec, Neilson et Cowan, 1831), 51 p., BRH, LII (1946) : 296s. 
Le Séminaire de Montréal publie, durant cette décennie, des cours abrégés de rhétorique en 1835 , et de belles-lettres en $1840^{20}$, manuels dont on ressentait vivement la nécessité. Ceux de rhétorique sont une synthèse de ce qu'on trouve alors de mieux sur le sujet. Ceux de belles-lettres sont inspirés du traité de Batteux ${ }^{21}$.

En mathématiques, professeurs et élèves travaillent dans les Institutions mathématiques de l'abbé Sauri ${ }^{22}$.

En 1836, Casimir Ladreyt, lance sur le marché une Nouvelle Arithmétique raisonnée ou cours complet de calcul théorique et pratique ${ }^{23}$. A partir de ce moment, Sauri ne sert qu'aux élèves de philosophie tandis que le volume de Ladreyt, plus facile, est réservé aux plus jeunes. Avec le temps, ces deux manuels disparaissent des pupitres des élèves. Comme les professeurs semblent souhaiter un manuel pensé par Sauri mais écrit par Ladreyt, ils choisissent celui de Jean-Antoine Bouthillier qui semble les satisfaire ${ }^{24}$.

La philosophie est une des dernières matières à s'enseigner dans un manuel. Le premier que nous trouvons, celui de l'abbé Jérôme Demers ${ }^{25}$, est un livre bien fait qui ne donne pas dans les erreurs du temps. Il cède la place vers 1850 à celui de Bouvier ${ }^{26}$ que des élèves utilisent encore en 1863.

Après cette énumération des manuels, nous donnerons un aperçu des livres de lectures. Ce genre d'imprimés arrive à un rythme beaucoup plus accéléré que les manuels car les dons de livres sont fréquents et les bibliothèques privées comportent généralement une forte quantité de livres de lecture. Voilà pourquoi les rayons de la bibliothèque du Séminaire de Nicolet sont

${ }^{20}$ Les auteurs de ces deux manuels sont inconnus. Cours abrégés de belles-lettres (Montréal, Leclerc et Jones, 1835), $260 \mathrm{p}$. ments.

21 C'est dans la préface de ces manuels que nous trouvons ces renseigne-

22 Directeur à l'évêque, 1804-1816, ASN, I: 313; Ibid., 1816-1839, II : 201, 482; ASN, Séminaire, I: 66 à 75; - (Paris, Ruault, 1774, Bachelier, $1835), 480 \mathrm{p}$.

23 (Montréal, s.e., 1836), $120 \mathrm{p}$.

24 Traité d'arithmétique (Montréal, J. B. Rolland et Fils, 1829), 180 p.; (Québec, Aug. Côté et Cie, 1855), 169 p., BRH, LIII (1946): 297s.

25 Institutiones philosophicae (Québec, Cary et Cie, 1835), 395 p.

26 Institutiones philosophicorum ad usum seminariorum et collegiarum (Paris, Lyon, 1824), VIII-281 p. 
chargés de 10,000 volumes en $1850^{27}$. Les livres à caractère religieux sont les plus nombreux dans une proportion de près de $75 \%$. L'abbé Laflèche ajoute à cela les livres profanes qui sont les plus recommandables au point de vue moral surtout.

Durant les dernières années de la période que nous sommes à étudier, le même abbé Laflèche se met en peine de dévoiler aux élèves les beautés de l'astronomie. Nous croyons qu'il a pu s'engager dans cette entreprise en se servant des instruments scientifiques achetés par les abbés Harper ${ }^{28}$ et Holmes ${ }^{29}$ entre 1830 et 1840. L'abbé Laflèche réussit enfin à obtenir un local spécial pour loger ces instruments à l'aide desquels il construit un planétaire qui fait l'orgueil du collège ${ }^{30}$.

\section{Activités parascolaires}

On constate, chez les éducateurs nicolétains, un désir non équivoque de suivre les traces de leurs prédécesseurs dans les cadres de la vie collégiale et dans les études. Cette orientation apparaît également dans les activités parascolaires. C'est le cas par exemple des travaux horticoles qui, à Nicolet comme en France ${ }^{31}$ et dans les autres collèges canadiens ${ }^{32}$, occupent alors les moments libres des élèves. Nous traiterons ensuite de l'Académie et de la Congrégation ${ }^{33}$.

L'occasion qui met en branle les travaux horticoles à Nicolet est la recherche de fleurs pour l'autel. L'abbé Leprohon profite en effet du besoin de parer l'autel pour donner à ses élèves le goût de cultiver les fleurs, de considérer leurs formes variées, leurs ramifications délicates, leur merveilleuse parure, enfin d'examiner leurs habitudes et leurs propriétés. L'abbé L.-Ed. Bois, à qui nous devons ces renseignements, précise que cette activité est à l'origine des travaux horticoles.

Il (l'abbé Leprohon) fit tant et si bien, dit l'abbé Bois, qu'il obtint aux écoliers d'abord un petit jardin, puis dans ce jardin un petit parterre à chacun, puis, enfin, des prix annuels aux plus dévoués, aux plus constants, aux plus laborieux. ${ }^{34}$

27 En 1850, le Séminaire de Montréal n'en avait pas plus. Maurault, op. cit., 84. Vers 1860, l'Université Laval en aurait eu 30,000.

$28 \mathrm{Mgr}$ Baillargeon à Harper, 1825-1848 (août 1831), ASN, I: 136.

29 ASN, Séminaire, V: 85-86.

30 Douville, op. cit., II : 2s.

31 Augustin Sicard, Les études classiques ..., 223s.

32 Maurault, op. cit., 60; Lebon, op. cit., I: 65.

33 Douville, op. cit., 303; Compaysé, op. cit., I : 179.

34 Bois, op. cit., 46s. 
L'abbé Leprohon ne travaille pas en vain car c'est de ce groupe de jeunes que fait partie le grand botaniste canadien, l'abbé Léon Provencher ${ }^{35}$. Elève à Nicolet de 1834 à 1840, il raconte dans ses souvenirs que le terrain n'étant pas aménagé à la campagne, il s'associe avec trois de ses confrères pour cultiver un carré à leur disposition et qu'il remporte presque chaque année le premier prix pour succès en horticulture ${ }^{36}$.

Avec les arbustes que plante l'abbé Pierre-Marie Mignault de 1807 à $1812^{37}$ et les travaux de l'abbé Ferland dans le bocage ${ }^{38}$, le Séminaire de Nicolet se présente à ceux qui l'habitent comme une oasis de paix et de verdure ${ }^{39}$, entretenue par les "hommes du bois" ${ }^{40}$ qui en prennent un soin jaloux.

Ces différentes occupations servent non seulement à "distraire l'esprit des occupations abstraites et sérieuses" ${ }^{41}$, mais aussi à développer l'émulation nécessaire à la bonne marche du collège, émulation assez bien entretenue jusque-là par les listes hebdomadaires ${ }^{42}$, par les examens de quartier et de fin d'année et par la distribution solennelle des prix ${ }^{43}$.

Parmi les activités parascolaires, il faut signaler particulièrement l'Académie et la Congrégation de la Sainte Vierge. La première, fondée en 1842 par l'abbé Ferland, Antoine Gérin-

35 Douville, op. cit., II: 155; Provencher peut s'écrire avec un a ou un e, nous dit Pierre-Georges Roy dans Toutes petites choses du régime anglais (Québec, 1946), II: 206. 15.

36 V.-A. Huard, La vie et l'œuvre de l'abbé Provencher (Paris, 1926),

37 [Robert Walsh], Le Séminaire de Nicolet (Montréal, 1867), 13.

38 Douville, op. cit., I: 32 ; le bocage est la forêt de pins qui se trouve à quelques centaines de pieds du collège.

39 J.-B. Meilleur, Mémorial de l'éducation du Bas-Canada (Québec, 1876), 76.

40 C'était le nom donné aux élèves chargés de nettoyer le bois et qui pouvaient fumer à cette occasion.

41 Meilleur, op. cit., 76.

42 Les premières listes qui existent datent de 1824 et il nous a semblé que par la suite il y eut toujours de telles listes.

43 L'année était divisée, durant cette période, en quartiers au nombre de trois par an et à chaque fin de quartier il y avait des examens. Les vacances ne durant qu'un mois et demi, l'année scolaire était fort longue; c'est pourquoi le travail scolaire était réparti en trois cycles au lieu de deux, comme aujourd'hui. Les examens étaient, jusqu'en 1858, faits en public et n'importe qui pouvait y assister car ils étaient annoncés dans les journaux. Pour plus de détails, voir Douville, op. cit., I: 234ss; AAQ, Séminaire de Nicolet, C-28, C-54. 
Lajoie ${ }^{44}$ et quelques autres, initie les membres à la discussion et à l'élocution. ${ }^{45}$ Les membres doivent présenter régulièrement un travail et les sujets choisis sont très variés. La renommée de l'Académie s'étend dans d'autres institutions. A Terrebonne ${ }^{46}$ et à Québec ${ }^{47}$ des sociétés demandent aux académiciens de Nicolet la constitution de leur mouvement.

La Congrégation de la Sainte Vierge, après une existence éphémère de 1806 à $1808^{48}$, est vraiment mise sur pied en $1823^{49}$. Elle tente de donner une note chrétienne à tous les actes de la vie des élèves et elle s'efforce principalement de maintenir la bonne conduite par des exercices religieux.

\section{Professeurs et élèves}

Toutefois, comme la valeur attachée à un cours d'études ne dépend pas uniquement de la qualité du matériel utilisé par les professeurs et les élèves, mais aussi des connaissances des professeurs et de la qualité intellectuelle des élèves, il importe de dire quelques mots des professeurs et des élèves, car ce sont des éléments importants à considérer pour bien apprécier la valeur d'un cours.

Les professeurs de ce temps-là se forment uniquement par la pratique de l'enseignement et par le travail personnel. Nous ne trouvons qu'une exception: celle de l'abbé François-Lesieur Désaulniers qui va chez les Jésuites à Georgetown en 1833-34 ${ }^{60}$. Les enseignants viennent presque tous du collège où ils ont fait leurs études classiques. A la fin de ces dernières, s'ils croient être appelés à devenir prêtres, ils revêtent la soutane, deviennent professeurs principaux en sixième ou en cinquième et suivent leurs élèves d'année en année.

Pendant ce temps, ils étudient la théologie. Quand l'évêque, après avoir pris information auprès du supérieur et du directeur, juge que l'un de ces professeurs-séminaristes a les qualifications voulues pour être prêtre, il lui donne l'onction sacerdotale. Ce

44 Douville, op. cit., I: 300.

45 Registre de l'Académie, I : 1s.

46 Ibid.: I: 300.

47 Ibid.: I : 13.

48 Registre de la Congrégation, I. Les archives de cette première congrégation comprennent des feuilles détachées au début de ce registre.

49 Ibid.: I: 1.

50 Douville, op. cit., I: 21. 
jeune prêtre est-il une personnalité supérieure ? Aussitôt l'évêque le nomme curé d'une paroisse. De cette manière, le collège de Nicolet ne peut compter que sur un personnel dont la valeur pédagogique laisse assez souvent à désirer ${ }^{51}$.

Un tel régime est peu favorable à une amélioration des cours et à la venue des professeurs laïcs. Le manque d'argent et peutêtre aussi une certaine crainte ${ }^{52}$ obligent les directeurs de cette maison à choisir minutieusement les rares candidats laïcs qui se présentent. Le premier dont il soit fait mention, un certain Matyou (Matthew), offre ses services comme professeur d'anglais ${ }^{53}$. L'abbé Leprohon est prêt à l'engager ${ }^{54}$, mais des difficultés dont nous ignorons la nature retardent la venue du premier professeur laïc jusqu'en 1833, date de la nomination de JosephVideu Hunt, comme professeur de musique ${ }^{55}$. Par la suite, quelques autres laïcs agissent comme professeurs auxiliaires. Voici leurs noms et leurs spécialités:

Evariste Tremblay, musique, 1848-1849.

John Harkin, anglais, 1851-1852.

Patrick Curran, dessin et anglais, 1855-1857.

Edmond-Octave Hardy-Chatillon, musique, après 1862 b6.

Qu'ils soient laïcs ou prêtres, les professeurs changent bien souvent et cela durant toute la période que nous étudions comme nous l'apprennent ces quelques lignes:

Quels que soient les professeurs qui nous viennent d'ailleurs, il est clair que les établissements qui nous les procurent ont eu le premier choix; et nous, ce sont nos meilleurs que nous perdons, de sorte que tout va en déclinant ${ }^{57}$.

Heureusement le problème va en s'atténuant quelque peu à la fin de la période que nous étudions. Les élèves sont les premiers à en profiter car jusque-là il arrive fréquemment que plusieurs ne terminent pas leur cours. Ceux qui y parviennent doivent travailler très fort.

51 Ibid., II: 1-126. Dans ces pages, se trouve une liste de tous les professeurs qui ont enseigné de 1803 à 1903. A parcourir cette liste, on peut se rendre compte de l'évidence d'un pareil énoncé.

52 Labarrère-Paulé, op. cit., ce travail fourmille de renseignements sur ce problème.

53 ASN, Séminaire, V: 4.

54 Ibid.

55 Douville, op. cit., II : $26 \mathrm{~s}$.

56 Ibid., II : 44, 45, 47, 48, 52, 53, 64 et 65 .

67 Ibid., I: 313. 
D'autre part, le fait que plusieurs élèves viennent d'un milieu rural assez difficilement touché par l'instruction ${ }^{58}$ a pour effet de peupler le collège d'adolescents qui n'ont même pas fait un cours primaire. De ces jeunes gens intéressés à faire leurs études classiques, plus de $90 \%$ sont de la campagne. La plupart habitent Nicolet et les paroisses environnantes. Un certain nombre sont originaires des paroisses du nord du fleuve où l'ancienneté du peuplement et une meilleure organisation paroissiale maintiennent une persévérance plus élevée que chez les élèves de tout autre endroit.

Parmi ceux qui complètent leur cours à Nicolet et qui choisissent une profession, la liste des carrières qui s'offre à eux est restreinte. Un grand nombre deviennent ${ }^{59}$ prêtres séculiers ${ }^{60}$. Les autres s'orientent en proportion assez égale vers la médecine et le droit. Quant à ceux qui quittent le collège avant la fin de leur cours, nous les retrouvons de temps à autre commerçants ${ }^{61}$ et le plus souvent cultivateurs. Parmi les anciens qui deviennent prêtres, nous en voyons qui sont évêques ${ }^{62}$, vicaires généraux, historiens ${ }^{63}$, botanistes ${ }^{64}$ et professeurs. Chez les laïcs, il $\mathrm{y}$ a, outre les médecins, les avocats et les notaires, des politiciens ${ }^{85}$, des écrivains et des journalistes ${ }^{66}$.

A lire la liste des professions que nous venons de donner, nous devinons que les besoins de cette période ne sont pas ceux d'aujourd'hui. Un collège qui s'occupe alors de former des jeunes qui puissent servir dans les professions libérales, la prêtrise, le commerce et même l'agriculture suffit à donner au groupe

58 Groulx, op. cit., I: 242.

59 Les chiffres que nous avons compilés, bien qu'incomplets, nous portent à croire qu'il en a été ainsi. Douville, op. cit., II : 127-202.

${ }_{60}$ Durant cette période, s'il y eut parmi les anciens des membres du clergé régulier, ils furent très peu nombreux.

${ }^{61} \mathrm{La}$ plupart deviennent marchands; très peu seront des financiers.

62 Mgr Joseph-Norbert Provencher, Mgr Charles-François Baillargeon, Mgr Thomas Cooke, Mgr Louis-François Richer-Laflèche, Mgr Jean-Charles Prince, Mgr Ignace Bourget.

63 L'abbé Jean-Baptiste-Antoine Ferland.

64 L'abbé Léon Provencher.

65 Antoine-Aimé Dorion fut sûrement le plus célèbre.

${ }^{66}$ Antoine Gérin-Lajoie, Etienne Parent, Arthur Buies, Louis Fréchette, Joseph-Guillaume Barthe. En 1866, Joseph Royal écrit: "de nos jours, la presse n'appartient-elle pas presqu'exclusivement à des Nicolétains? Vois: la Minerve, de Montréal, le Canada, d'Ottawa, le Journal de TroisRivières, la Gazette de Sorel, le Journal de Lévis, le Foyer canadien de Québec, sont tous des journaux rédigés ou dirigés par des élèves (anciens) de Nicolet." Revue Canadienne (1886) : 377. 
canadien-français les hommes dont il a besoin. Le Séminaire de Nicolet est ainsi en accord avec les désirs de son siècle. Ce collège se place par le fait même dans la grande tradition classique qui ne voyait pas, pour arriver à ce but, d'autre moyen qu'une discipline sévère. Une telle discipline, pense-t-on alors, est la gardienne toute choisie des valeurs de cette société plutôt autarcique. N'allons pas oublier toutefois que cette maison a pour fonction de donner au monde des chefs, et des chefs à la foi forte. D'où l'importance que les éducateurs nicolétains accordent à des sociétés comme l'Académie et la Congrégation. Les résultats ne paraissent pas des plus brillants, à nos yeux d'hommes du $\mathrm{XX}^{\mathrm{e}}$ siècle. Pour les gens de la première moitié du XIX $\mathrm{XI}^{\mathrm{e}}$ siècle cependant, l'œuvre réalisée par les éducateurs de ce collège est remarquable puisque de cette institution sortent des jeunes gens qui, une fois devenus adultes, deviennent les chefs de file de la nation canadienne-française comme prêtres, politiciens, écrivains et journalistes. 